\title{
Transport through Single-Wall Carbon Nanotubes Weakly Coupled to External Leads
}

\author{
I. WeYMANN ${ }^{a, b}$, S. KROMPIEWSKI ${ }^{c}$ AND J. BARNAŚ ${ }^{a, c}$ \\ ${ }^{a}$ Department of Physics, Adam Mickiewicz University \\ Umultowska 85, 61-614 Poznań, Poland \\ ${ }^{b}$ Theoretical Physics Department \\ Budapest University of Technology and Economics \\ H-1521 Budapest, Hungary \\ ${ }^{c}$ Institute of Molecular Physics, Polish Academy of Sciences \\ M. Smoluchowskiego 17, 60-179 Poznań, Poland
}

\begin{abstract}
We consider transport properties of single-wall metallic carbon nanotubes weakly coupled to external leads. In particular, we analyze the conductance and shot noise of nanotubes coupled to nonmagnetic leads and show that the shot noise may become super-Poissonian depending on the ground state of the nanotube. In addition, we also show that when the nanotube is coupled to one ferromagnetic and one nonmagnetic lead, it can operate as a gate-controlled spin diode.
\end{abstract}

PACS numbers: 72.25.Mk, 73.63.Kv, 85.75.-d, 73.23.Hk

\section{Introduction}

Recent progress in nanotechnology has enabled both the investigation of different quantum phenomena at the nanoscale as well as the manipulation of single charges and their spins. Among other structures, carbon nanotubes have proved to be ideal devices for studying the quantum behavior in one and zero dimensions. This is associated with their extreme flexibility - they can be either metallic or semiconducting $[1,2]$. Furthermore, depending on the quality of the coupling to external leads, they can behave as electron waveguides, display the Kondo physics or exhibit the Coulomb charging effects characteristic of quantum dots [3]. In addition, when coupled to ferromagnetic leads, they show pronounced magnetoresistance effects [4].

In this paper we analyze transport properties of single-wall metallic carbon nanotubes (CNT) weakly coupled to external leads. Hamiltonian of the system has the general form, $H=H_{\mathrm{L}}+H_{\mathrm{R}}+H_{\mathrm{CNT}}+H_{\mathrm{T}}$. The first two terms describe noninteracting electrons in the leads, $H_{r}=\sum_{\boldsymbol{k} \sigma} \varepsilon_{r \boldsymbol{k} \sigma} c_{r \boldsymbol{k} \sigma}^{\dagger} c_{r \boldsymbol{k} \sigma}$, for the left $(r=\mathrm{L})$ and right $(r=\mathrm{R})$ leads, with $\varepsilon_{r \boldsymbol{k} \sigma}$ being the energy of an electron with the wave vector $\boldsymbol{k}$ and spin $\sigma$ in the lead $r$. The nanotube is described by [5]:

$$
H_{\mathrm{CNT}}=\sum_{\mu j \sigma} \varepsilon_{\mu j} n_{\mu j \sigma}+\frac{U}{2}\left(N-N_{0}\right)^{2}
$$

$$
+\delta U \sum_{\mu j} n_{\mu j \uparrow} n_{\mu j \downarrow}+J \sum_{\mu j, \mu^{\prime} j^{\prime}} n_{\mu j \uparrow} n_{\mu^{\prime} j^{\prime} \downarrow},
$$

where $N=\sum_{\mu j \sigma} n_{\mu j \sigma}$, and $n_{\mu j \sigma}=d_{\mu j \sigma}^{\dagger} d_{\mu j \sigma}$. The $j$-th discrete energy level in the subband $\mu(\mu=1,2)$, $\varepsilon_{\mu j}$, is given by $\varepsilon_{\mu j}=j \Delta+(\mu-1) \delta$, where $\Delta$ is the spacing between levels following from quantization in a particular subband, and $\delta$ is the energy mismatch between the level sets corresponding to the two subbands. $U$ denotes the charging energy of the nanotube, $\delta U$ is the on-level Coulomb correlation, $N_{0}$ is the charge induced by the gate voltage, while $J$ is the Stoner exchange parameter. The tunneling processes between the CNT and electrodes are described by the Hamiltonian $H_{\mathrm{T}}=\sum_{r=\mathrm{L}, \mathrm{R}} \sum_{\boldsymbol{k}} \sum_{\mu j \sigma}\left(t_{r j} c_{r \boldsymbol{k} \sigma}^{\dagger} d_{\mu j \sigma}+t_{r j}^{*} d_{\mu j \sigma}^{\dagger} c_{r \boldsymbol{k} \sigma}\right)$, where $t_{r j}$ stands for the tunnel matrix element between the lead $r$ and the $j$-th level. Coupling of the $j$-th level to external leads is given by $\Gamma_{r j}^{+(-)}=\left(1 \pm p_{r}\right) \Gamma / 2$, for the majority (minority) electrons, where $p_{r}$ is the spin polarization of lead $r$. In the following we assume $\Gamma_{r j}=\Gamma / 2$ for all values of $j$ and $r$.

When the nanotube is weakly coupled to external leads, for bias voltages exceeding a threshold voltage, the current flows due to the sequential tunneling processes. On the other hand, below the threshold voltage the first-order tunneling is exponentially suppressed due to the Coulomb interaction, leading to the Coulomb blockade. In this transport regime, the current is mediated by second-order processes (cotunneling) which in- 
volve correlated tunneling through virtual states of the nanotube. In order to determine transport properties of single-wall metallic carbon nanotubes in the sequential and cotunneling regimes, we employ the real-time diagrammatic technique [6-8]. It consists in a systematic expansion of the nanotube density matrix and the current operator with respect to the coupling strength $\Gamma$. Time evolution of the density matrix can be visualized as a sequence of irreducible self-energy blocks, $W_{\chi \chi^{\prime}}$, on the Keldysh contour. The matrix elements $W_{\chi \chi^{\prime}}$ describe transitions between the many-body states $|\chi\rangle$ and $\left|\chi^{\prime}\right\rangle$ of the nanotube. Within the real-time diagrammatic approach, the nanotube occupation probabilities can be found from [7], $\left(\tilde{\boldsymbol{W}} \boldsymbol{p}^{\text {st }}\right)_{\chi}=\Gamma \delta_{\chi \chi_{0}}$, where $\boldsymbol{p}^{\text {st }}$ is the vector containing probabilities and $\tilde{\boldsymbol{W}}$ is given by $\boldsymbol{W}$ with one arbitrary row $\chi_{0}$ replaced by $(\Gamma, \ldots, \Gamma)$ due to normalization, $\sum_{\chi} p_{\chi}^{\text {st }}=1$. On the other hand, the current flowing through the system is given by $I=(e / 2 \hbar) \operatorname{Tr}\left\{\boldsymbol{W}^{\mathrm{I}} \boldsymbol{p}^{\mathrm{st}}\right\}$, where the self-energy matrix $\boldsymbol{W}^{\mathrm{I}}$ is equal to the matrix $\boldsymbol{W}$ modified so as to account for a number of electrons transferred through the nanotube. In addition, we also calculate the Fano factor, $F=S /(2 e|I|)$, which describes the deviation of the shot noise from its Poissonian value, $S_{p}=2 e|I|$. The formula for the shot noise was derived in Ref. [7]. We further perform a perturbation expansion of the self-energy matrices and probabilities in the coupling $\Gamma, \boldsymbol{W}^{(\mathrm{I})}=\boldsymbol{W}^{(\mathrm{I})(1)}+\boldsymbol{W}^{(\mathrm{I})(2)}+\ldots$, and, $p^{\text {st }}=p^{\text {st(0) }}+p^{\text {st(1) }}+\ldots$, which allows us to determine the first-order (sequential) and second-order (cotunneling) transport properties.

It is now well-established that single-wall metallic carbon nanotubes exhibit the four-electron periodicity, which is a consequence of the two spin-degenerate subbands of the nanotube [3]. Furthermore, depending on the internal parameters of the nanotube, two different sequences of the ground states can be realized. The first one is $S=0,1 / 2,0,1 / 2$, whereas the second one is $S=0,1 / 2,1,1 / 2$, where $S$ is the total spin of the nanotube. The first scenario is realized when $\delta U+J<\delta$, whereas the second scenario happens for $\delta U+J>\delta$. In the case of first sequence, the shell filling of the nanotube is realized in this way that the next orbital levels are being occupied only after the lower lying levels are full. On the other hand, in the second scenario, in the ground state with two electrons on the nanotube, each electron occupies different orbital level which, due to the exchange interaction $J$, leads to the formation of a triplet state. Here, we focus on the sequence $S=0,1 / 2,1,1 / 2$ and merely note that the first one was considered elsewhere [9]. To model the carbon nanotube in most realistic way, we have taken the parameters derived from the experiments of Liang et al. [3].

\section{Results and discussion}

In Fig. 1 we show the bias and gate voltage dependence of the differential conductance (a) and the Fano factor (b) for single-wall carbon nanotube coupled to nonmagnetic leads. The noise in the small bias regime $\left(|e V| \leq k_{\mathrm{B}} T\right)$ is dominated by thermal Nyquist-Johnson noise, which leads to a divergence of the Fano factor as $V \rightarrow 0$. This is marked by the black thick line around $V=0$ in Fig. 1 b. The black diamonds in Fig. 1a correspond to blockade regions, where sequential transport is exponentially suppressed and current is dominated by the second-order processes. Furthermore, the four-electron periodicity is clearly visible in the linear conductance, while the additional lines in the conductance for voltages larger than the threshold for sequential tunneling are due to excited states participating in transport. Starting from the large Coulomb diamond, by sweeping the gate, the ground state of the nanotube changes as $S=0,1 / 2,1,1 / 2$. In the Coulomb blockade regime we find a super-Poissonian shot noise $(F>1)$ due to bunching of inelastic cotunneling processes through the nanotube. This is most visible in the cotunneling regime where the two nearest orbital levels of different subbands are fully occupied, $S=0$. The noise is also super-Poissonian in the Coulomb blockade where the ground state is a triplet, $S=1$, its value is however smaller than that for $S=0$. On the other hand, once the bias voltage is increased above the threshold and sequential processes start to dominate the current, the Fano factor becomes generally sub-Poissonian and for large bias voltages approaches $F=1 / 2$. This behavior is in qualitative agreement with recent experiments carried out by Onac et al. [10], who found super-Poissonian shot noise in the Coulomb blockade regime, whose magnitude depends on the ground state of the nanotube.

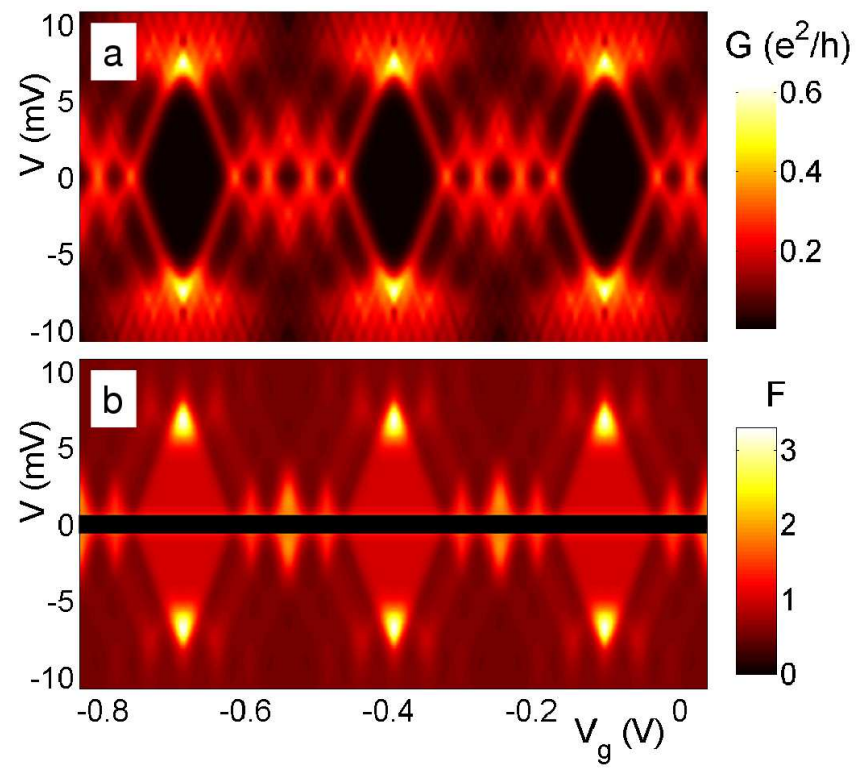

Fig. 1. Differential conductance (a) and Fano factor (b) as a function of bias and gate voltages. The parameters are: $\Delta=8.4 \mathrm{meV}, U / \Delta=0.26, J / \Delta=0.12$, $\delta U / \Delta=0.04, \delta / \Delta=0.1, k_{\mathrm{B}} T / \Delta=0.035, p_{\mathrm{L}}=p_{\mathrm{R}}=0$, $x=0.14$, and $\Gamma=0.2 \mathrm{meV}$. Here $x$ relates the gate voltage to the electrochemical potential shift. 


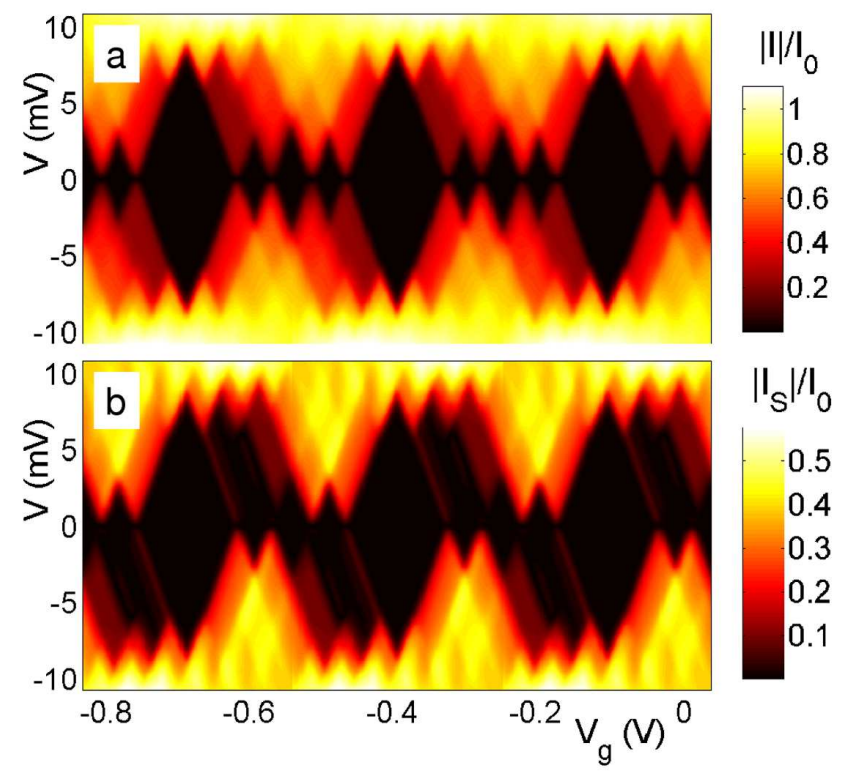

Fig. 2. The absolute value of the current $I$ (a) and the spin current $I_{\mathrm{S}}(\mathrm{b})$ as a function of bias and gate voltages for $p_{\mathrm{L}}=0, p_{\mathrm{R}}=0.7, k_{\mathrm{B}} T / \Delta=0.025$, and $I_{0}=e \Gamma / \hbar \approx 48.7 \mathrm{nA}$. The other parameters are the same as in Fig. 1.

Another interesting situation occurs when the nanotube is coupled to one magnetic and one ferromagnetic lead. In this case the nanotube can operate as a spin diode $[11,12]$. In Fig. 2 we show the density plots of absolute value of the current and the spin current. The dependence of differential conductance is qualitatively similar to that shown in Fig. 1a and is not shown here. Because the diode operates mainly in the sequential regime, in calculations we have taken into account only the first-order processes. As can be seen in the figures, transport characteristics are not symmetric with respect to the bias reversal. This is associated with the spin-dependence of tunneling between the nanotube and ferromagnetic electrode, which leads to increased rate for tunneling of the spin-majority electrons as compared to the spin-minority ones. On the other hand, the matrix elements for tunneling between the nonmagnetic lead and the nanotube do not depend on the spin orientation. Furthermore, it can be seen that for certain gate voltages the bias dependence of the current displays features typical of diodes the current is suppressed for one bias polarization and increased for another one. Due to the spin dependence of tunneling processes, charge current is associated with a nonzero spin current $I_{\mathrm{S}}=I_{\uparrow}-I_{\downarrow}$ flowing through the sys- tem, where $I_{\sigma}$ is the current flowing in the spin- $\sigma$ channel. Spin current, similarly as the charge current, also reveals features typical of spin-diode - it is increased for one bias polarization and suppressed for another one, see Fig. 2b. As a consequence, carbon nanotubes when coupled to one nonmagnetic and one ferromagnetic lead can be used as spin diodes, and their functionality can be controlled additionally by a gate voltage, see Fig. 2 . These observations are in qualitative agreement with very recent experiments performed on single-wall carbon nanotubes coupled to $\mathrm{Nb}$ and Co electrodes [12].

\section{Acknowledgments}

This work, as part of the European Science Foundation EUROCORES Programme SPINTRA, was supported by funds from the Ministry of Science and Higher Education as a research project in years 2006-2009 and by the EU grant CARDEQ under contract IST-021285-2. I.W. also acknowledges support from the Foundation for Polish Science.

\section{References}

[1] R. Saito, M.S. Dresselhaus, G. Dresselhaus, Physical Properties of Carbon Nanotubes, Imperial College Press, London 1998.

[2] M.P. Anantram, F. Leonard, Rep. Prog. Phys. 69, 507 (2006).

[3] W. Liang, M. Bockrath, H. Park, Phys. Rev. Lett. 88, 126801 (2002).

[4] S. Sahoo, T. Kontos, J. Furer, C. Hoffmann, M. Gräber, A. Cottet, C. Schönenberger, Nature Phys. 1, 102 (2005).

[5] Y. Oreg, K. Byczuk, B.I. Halperin, Phys. Rev. Lett. 85, 365 (2000).

[6] H. Schoeller, G. Schön, Phys. Rev. B 50, 18436 (1994); J. König, J. Schmid, H. Schoeller, G. Schön, Phys. Rev. B 54, 16820 (1996).

[7] A. Thielmann, M.H. Hettler, J. König, G. Schön, Phys. Rev. Lett. 95, 146806 (2005).

[8] I. Weymann, J. Barnaś, S. Krompiewski, Phys. Rev. B 76, 155408 (2007).

[9] I. Weymann, J. Barnaś, S. Krompiewski, condmat/0803.1969.

[10] E. Onac, F. Balestro, B. Trauzettel, C.F.J. Lodewijk, L.P. Kouwenhoven, Phys. Rev. Lett. 96, 026803 (2006).

[11] I. Weymann, J. Barnaś, Appl. Phys. Lett. 92, 103127 (2008).

[12] Ch.A. Merchant, N. Markovic, Phys. Rev. Lett. 100 , 156601 (2008). 